\title{
The Experiences of Palliative Care Specialists in Implementing Palliative Care into Their Work Settings in Lusaka, Zambia
}

\author{
Patience Mbozi ${ }^{1,2 *}{ }^{(D}$, Elizabeth Namukwaya ${ }^{3}$, Joseph Chaila ${ }^{4}$ \\ ${ }^{1}$ Chreso University, Lusaka, Zambia \\ ${ }^{2}$ Zambia Oncology Nurses Society, Lusaka, Zambia \\ ${ }^{3}$ Department of Medicine, College of Health Sciences, Makerere University, Kampala, Uganda \\ ${ }^{4}$ Ministry of Health, Lusaka, Zambia \\ Email: *patiencembozi@gmail.com
}

How to cite this paper: Mbozi, P., Namukwaya, E. and Chaila, J. (2019) The Experiences of Palliative Care Specialists in Implementing Palliative Care into Their Work Settings in Lusaka, Zambia. Health, 11, 1664-1683. https://doi.org/10.4236/health.2019.1112126

Received: November 21, 2019

Accepted: December 27, 2019

Published: December 30, 2019

Copyright (c) 2019 by author(s) and Scientific Research Publishing Inc. This work is licensed under the Creative Commons Attribution International License (CC BY 4.0).

http://creativecommons.org/licenses/by/4.0/

\section{cc) (i) Open Access}

\begin{abstract}
There is a significant unmet need for palliative care globally: of the 58 million people dying annually, it was estimated that at least $60 \%$ will have the prolonged advanced illness and dying and would benefit from palliative care. Zambia has a total population of 15,066,266 people. About 1,150,400 people are living with HIV and 18,900 people were reported to have died of HIV in 2014 alone. The most recent palliative care situational analysis in Zambia highlighted that palliative care remained "an essential absent factor" and revealed that there were gaps in the type of care given in various home based care $(\mathrm{HBC})$ programs. Therefore, this study aimed at exploring the experiences of trained palliative care specialists in implementing palliative care in Lusaka, Zambia. This study was an exploratory qualitative research. The population comprised of the trained palliative care specialists in either degree or diploma in palliative care, working in Lusaka. Purposive sampling was used and the sample totaled $6(n-6)$. The data collection was done using a semi-structured interview guide, through face-to-face in-depth interviews. The interviews lasted for an average of 20 minutes. Prompts and probes were used to increase detailed analysis explorations. Eleven themes broadly classified as facilitators or challenges emerged from the study. Among the facilitators: training influence; higher level support; effective team work and delivery models; and personal development and motivation to serve. challenges included: lack of PC knowledge; lack of essential medication for PC; work burden; lack of financial resources; lack of acceptance of PC; lack of PC guidelines; and legal restrictions on the use of opioids. The study provided evidence of the challenges and facilitators that influence palliative care that specialists experience while implementing PC. It is therefore, recommended that more research,
\end{abstract}


education, effective teamwork is promoted as the study revealed that participants needed support for effective implementation of palliative care.

\section{Keywords}

Palliative Care, Facilitators, Challenges, Palliative Care Specialist, Palliative Care Implementation

\section{Introduction}

World Health Organization (2014), states that good palliative care improves the quality of life of patients with life-threatening illnesses and their families [1]. Stjernsward, et al. (2007) reported that, there was a significant unmet need for palliative care globally: of the 58 million people dying annually, at least $60 \%$ (35 million) will have the prolonged advanced illness and dying and would benefit from palliative care [2].

The World Health Organizations' (WHO) four fundamental pillars for palliative care access include: government policy to improve access, education of health workers and the public, drug availability and implementation throughout health-care systems. Logie (2012) reported palliative care services having existed in Zambia since 1997 but later closed due to funding cuts hence, leading to a critical barrier to the support of palliative care in Zambia and also to improving access for all [3]. In the same way, Goma et al. (2008) reported that Zambia was lacking in all the four aforementioned pillars of establishing palliative care in the country [4].

Palliative care specialists have a huge role in the implementation of palliative care (Street and Blackford, 2001) [5]. They provide leadership to others so that other clinicians observe and incorporate the practice into their care (Street and Blackford, 2001; Murray et al. 2008) [5] [6]. Therefore, palliative care implementation includes services such as: symptom control, decision making, management of treatment complications, communication, psychosocial care, and coordination of care (Becker et al. 2011; Kulkarni, 2011) [7] [8].

The Central Intelligence Agency (2014) reported that Zambia has a total population of 15,066,266 people with $12.37 \%$ HIV prevalence rate and $7.2 \%$ diabetes prevalence rate [9]. About 1,150,400 people are living with HIV and 18,900 people were reported to have died of HIV in 2014 alone [9]. Cancer diseases Hospital (2014) annual report showed a steady increase in the number of cancer patients accessing cancer services from 1800 in 2013 to 2250 in 2014 [10].

There is no current comprehensive palliative care situational analysis or mapping of services in Zambia. The most recent was in 2008 by Goma et al. (2008) who highlighted that palliative care remained "an essential absent factor" and revealed that there were gaps in the type of care given in various home based care (HBC) programs [4]. They describe late presentation of patients compounded by difficult access to medical facilities. 
Moreover, anecdotal data has shown that there is a great lack of enthusiasm in the implementation of palliative care among palliative care specialists. So far, no research has been done to determine their experiences. Moreover, several people have been trained but this has not been translated into practice. Therefore, this research seeks to establish the reason why this is happening to further inform practice.

This research therefore seeks to establish information on the experiences of specialist palliative care providers in implementing palliative care. The Knowledge of these experiences will enable palliative care specialists to identify good implementation strategies for palliative care. Further, the study will provide evidence on how best palliative care can be integrated in various health care settings. The research may also provide stimulus to new research capacity building.

\subsection{Research Question}

What are the experiences of palliative care specialists in the implementation of palliative care in their work settings?

\subsection{Study Objective}

This study aims to explore the experiences of trained palliative care specialists in implementing palliative care in Lusaka, Zambia.

\section{Literature Review}

A PUBMED and google scholar database search was performed using keywords "palliative care", "palliative care experience", "palliative care implementation", "hospice OR hospital", "palliative care specialist", “Africa” and "Zambia” limited to English language from 2000 to 2015. Several papers were identified but only 10 were chosen as they specifically discussed the experiences of palliative care specialists in implementing palliative care in detail.

The literature showed that there were challenges and facilitators to the implementation of palliative care services. The challenges mentioned included; lack of Government policy, Cancer and HIV Burden; Lack of palliative care education; lack of resources in the implementation of palliative care; challenging cultural beliefs; and unavailability of opioids and myths about opioid use (Onyeka, 2011; Ntizimira et al. 2014; Clark et al. 2007; Grant et al. 2011; Logie, 2012; Basu, 2013; McDermott, 2008; Hasson et al. 2008; Brazil et al. 2010) [3] [11]-[18]. The facilitators included; good management support and growing public awareness of palliative care (Hasson et al. 2008; Brazil et al. 2010) and availability and affordability of a full multidisciplinary team (Clark et al. 2007; Hasson et al. 2008; Brazil et al. 2010) [13] [17] [18] [19] [20]. Therefore, this literature review will be critically discussed on the palliative care implementation challenges and facilitators noted from the papers while comparing and contrasting their views.

\subsection{Lack of Government Policy}

Stjernsward et al. (2007) suggested that Governments in developing countries needed to include palliative care in the National Health Plan and devise a me- 
chanism for funding and/or service delivery models that support service delivery [2]. Unfortunately, most countries in Africa including Zambia have not yet included palliative care in their national policies or regulations Ndungu 2011; Mwangi-Powell, 2011; Rawlinson et al. 2014; Logie, 2012; Goma et al. 2008; Kalolo, 2010) [3] [4] [19] [20] [21] [22].

According to Hill (1993) Policy is a broad statement reflecting future goals and aspirations and provides guidelines for carrying out those goals [23]. Onyeka (2011) states that overwhelming numbers of patients and being frequently called to see patients in other hospitals negatively affected their services; this happened due to lack of human and social resources resulting from palliative care not recognized and prioritized by the policy makers [11]. Studies show that lack of national palliative care policy hindered effective implementation of palliative care services; practitioners could not practice it despite having relevant knowledge (McDermott, 2008; Clark et al. 2007) [13] [16]. Therefore, lack of palliative care inclusion in the national health policy negatively affects palliative care implementation.

\subsection{Huge Burden of Cancer and HIV}

Onyeka (2011) reported that due to the cancer and HIV burden coupled with the late presentations and late diagnosis of patients, they could not meet up to the overwhelming demands of patients needing palliative care and this caused some patients to miss the care [11]. Similarly, Grant et al. (2011) mentioned late presentations and increasing numbers of patients in need of palliative care as hindrances to palliative care provision [14]. McDermott, (2008) stipulated that high density population across huge geographical areas with majority of people living in impoverished circumstances had proved a difficult barrier to provide effective palliative care services in India [16].

\subsection{Lack of Palliative Care Education}

WHO (2014) recommended that "governments should ensure that healthcare workers are adequately trained in palliative care and the relief of cancer pain" [1]. WHO (2014) further reported that, the majority of health professionals, worldwide, had little or no knowledge of the principles and practices of palliative care [1]. Lack of palliative care training and its absence in the medical curriculum has impacted negatively on effective implementation of palliative care services (McDermott 2008; Clark et al. 2007; Brazil et al. 2010) [13] [16] [18]. Onyeka (2011) highlighted the combination of lack of trained professionals in palliative care and an ever-increasing patient load having a negative impact in their implementation of palliative care [11]. Further, McDermott (2008) research revealed that lack or little education about morphine had hindered effective implementation of palliative care in India [16].

\subsection{Lack of Resources in the Implementation of Palliative Care}

Merriman (1999) mentioned that palliative care services in developing countries 
needed to be delivered in a way that met the cultural, spiritual and economic needs of the people [24]. According to Stjernsward et al. (2007) Implementation of palliative care required sufficient funding and appropriate service-delivery models to be put in place [2].

Brazil et al. (2010) highlighted that inadequate resources to support patient care and lack of continuity of care via timely sharing of patient information hindered effective palliative care implementation [18]. Similarly, Onyeka (2011) stated that lack of patient referral could have been caused by insufficient resources, lack of awareness (healthcare workers and the public), cultural and religious beliefs which resulted in a number of patients missing palliative care services and this made implementation of palliative care strenuous and difficult [11]. Moreover, McDermott (2008) added that insufficient funding for the palliative care services and the absence of a state-sponsored social security system or effective medical insurance scheme has made it difficult to implement palliative care [16].

\subsection{Challenging Cultural Beliefs}

Onyeka (2011) reported that speaking of an impending death in Nigeria is a "taboo", because of the culture of "death denial" among patients, their relatives, and healthcare professionals [11]. Similarly, it was been reported that clinicians were reluctant to disclose unfavorable prognosis and therefore ended up prescribing less than adequate analgesia because of opiophobia, opioignorance and family expectations (Yanjun et al. 2010; Brazil et al. 2010; McDermott 2008) [16] [18] [25]. Furthermore, Hasson et al. (2008) reported that communication and cultural differences amongst staff had hindered provision of quality palliative care [17]. Similary, Brazil et al. (2010) postulated that respecting the patient autonomy even when they were wrong hindered provision of appropriate care, effective pain management, and giving information to patients or family members [18].

\subsection{Unavailability of Opioids and Myths about Opioid Use}

African Palliative Care Association (2010) advocates that access to palliative care medicines is crucial for ensuring effective pain and symptom management; they added that increasing access involves improving supply, legislation, education, distribution and increasing prescribers [26].

McDermott (2008) mentioned opioid accessibility as a constant frustrating problem for the clinicians in India [16]. Clark et al. (2007) reported that morphine unavailability in Africa, lack of experience in prescribing morphine, fear of opioid side effects and few dispensing services of opioids have negatively affected palliative care implementation [13]. Onyeka (2011) argued that opioid unavailability and opiophobia are very prevalent in their practice and therefore, recommended that policymakers should workout national guidelines pertaining to the availability, reasonable cost, prescription, and safe storage of opioids as this would enable effective palliative care implementation [11]. 


\subsection{Good Management Support and Growing Public Awareness of Palliative Care}

Hasson et al. (2008) revealed one key factor which led to success of their palliative care services and this was management support [17]. Additionally, McDermott (2008) reported that growing public and medical acceptance of palliative care, had resulted in increasing number of volunteers, and the ongoing partnership with international support agencies [16]. Moreover, Hasson et al. (2008) added on that access to resources made palliative care implementation interesting [17]. Clark et al. (2007) further asserted that despite palliative care policy not being officially sanctioned by the government of Kenya, there had been significant palliative care recognition, support and appreciation by the Ministry of Health-Kenya [13].

\subsection{Availability and Affordability of a Full Multidisciplinary Team}

Hasson et al. (2008) highlighted that access to resources and payment for palliative care specialists who have worked outside normal working hours encouraged the effective implementation of palliative care [17]. Similarly, Clark et al. (2007) highlighted that the complete multidisciplinary team allowed practitioners in South Africa to offer quality palliative care services and support other departments that needed the services [13]. Hasson et al. (2008) further reported that flexibility and availability of the supervisors for advice encouraged palliative care implementation [17].

\subsection{Conclusion}

The literature review revealed different experiences of HCWs while implementing PC. The facilitators included: Good management support and growing public awareness of PC, availability and affordability of a full multi-disciplinary team whilst challenges included: Lack of government policy, cancer and HIV burden, lack of PC education, lack of resources in the implementation of PC, challenging cultural beliefs and unavailability and myths of opioids.

\subsection{Methodology}

The study setting was in Lusaka, Zambia and targeted 14 palliative care specialists with Diplomas and Degrees in palliative care who were working in the University Teaching Hospital, Cancer Hospital and Kalingalinga Clinic, and were willing to participate in the study. Purposive sampling was used and 6 of the specialists consented and participated. Data collection was done using a semi-structured interview guide, through face-to-face in-depth interviews in order to obtain greater richness of data for the study. This enabled the researcher to lead, probe and remain in control of the interview and ensured that participants understood the questions asked. The interview guide had been peer reviewed with a nurse colleague in order to check its appropriateness and the interviews were audio recorded. Prompts and probes were used to increase detailed analysis explorations 
(Williman, 2011 and Becker et al.) [7] [27].

Interviews lasted 20 minutes on average. Data collection and analysis was done concurrently. The analysis began immediately after the first interview and new themes were added as more data was collected. Data analysis involved transcription of the audio recorded interviews where the themes were coded into categories. Additionally, the analysis occurred and continued until no further new information could be gathered. The transcriptions were revised several times to identify new themes. Themes were then developed and analysed (Aveyard, 2010) [23]. These themes were applied to the research question and enabled the formulation of a conclusion.

Permission to conduct the study was obtained from the ethics committee of the Ministry of Health in Zambia and from relevant managers prior to commencement of the research. Informed consent and consent to audio tape the interviews were obtained from the study participants. Interviews were conducted in a private room. After the transcription, the interviews were transferred to a $\mathrm{CD}$ and deleted from the computer. All data sheets and the $\mathrm{CD}$ were sealed in an envelope and placed in a safe; to be kept for a period of three years after the report has been published thereafter will be destroyed.

\section{Results}

\subsection{Participants' Characteristics}

The age of the participants ranged from 30 to 54 years comprising of 3 females and 3 males. Five had completed a diploma in palliative care while one was still on training. By profession, 3 were medical doctors while the other three were registered nurses (Table 1).

\subsection{Themes Arising from the Data}

Five themes arose from the study, which were broadly classified as facilitators and challenges/barriers in implementing PC.

\subsection{Facilitators}

The themes identified under facilitators were: Training influence; higher level support; Effective team work and delivery models; Personal development and motivation to serve.

\subsection{Training Influence}

All the respondents mentioned that the training was important as it reduced the knowledge gap. They used terms such as the training was "good" and "useful" in describing it which widened and enhanced their knowledge in palliative care.

"I think it was, haaa, it was good because it, it introduced, haaa, me to, to the concept of palliative care and went ahead to, haaa, give me an understanding on how I can implement and monitor, haaa, the implementation of palliative care within any setting, be it in a hospital or a community or a hospice setup and also it, eh, 
Table 1. Demographic information of the participants $(n=6)$.

\begin{tabular}{|c|c|}
\hline Characteristics & n \\
\hline \multicolumn{2}{|l|}{ Age Groups } \\
\hline $30-34$ & 2 \\
\hline $35-39$ & 2 \\
\hline $50-54$ & 2 \\
\hline \multicolumn{2}{|l|}{ Sex } \\
\hline Male & 3 \\
\hline Female & 3 \\
\hline \multicolumn{2}{|l|}{ Training } \\
\hline Diploma in Palliative Care & 6 \\
\hline Completed & 5 \\
\hline Currently in Training & 1 \\
\hline \multicolumn{2}{|l|}{ Profession } \\
\hline Medical Doctor & 3 \\
\hline Registered Nurse & 3 \\
\hline \multicolumn{2}{|l|}{ Years of Experience } \\
\hline $0-1$ years & 2 \\
\hline $1-2$ years & 2 \\
\hline $2-3$ years & 0 \\
\hline 3 - 4 years & 2 \\
\hline \multicolumn{2}{|l|}{ Institution } \\
\hline Cancer Diseases Hospital & 1 \\
\hline Kalingalinga First Level Hospital & 1 \\
\hline University Teaching Hospital & 4 \\
\hline
\end{tabular}

it gave me the much needed knowledge on how to prescribe opioid..." (Respondent 1$)$.

"I think it was useful, haaa, it introduced me to new things about palliative care. And, haaa, it was a good opportunity to see how, haaa, palliative care is been conducted in other places." (Respondent 4).

"It's a good training, it equips health workers to deal with issues that surround palliative care and it's a good solid base for someone to get into palliative care as a specialty." (Respondent 6).

\subsection{Higher Level Support}

Creation of palliative care positions in the civil service was recognised and noted by the respondents as they highlighted that it was an indication that palliative care was being recognised as a specialty in Zambia.

"...lack of, haaa, the service having been incorporated into the civil service, only recently has it been introduced. So in the past it has been very difficult to 
even talk about palliative care, with an establishment, it becomes much easier for people to practice..." (Respondent 6).

Further, management support at institutional level was reported to have made palliative care implementation easier and feasible. This support was a motivating factor as highlighted below.

"The hospital management as I said earlier on, they were very very supportive. And everything that we did as a palliative care team, every financial request, haaa... that we requested from them, they were able to provide." (Respondent 1)

"Well, the management, the top management at the hospital, especially the SMS (senior medical superintendent) was very very supportive." (Respondent 3 ).

And

“... Cause when we review a patient, you know there is this resentment about a nurse who is maybe a palliative care consultant writing notes in the patient's records. They resented a bit, but they were told by the SMS that we can respond to their consultations and the only way we can interact with them was to put up the notes in the file so that they can follow-up and discuss..." (Respondent 3).

“...Like I mentioned management level they have given us the opportunity to talk to them and we are able to approach them, haaa, when have things that we need to help us move the programme forward." (Respondent 4).

"In terms of management support, the UTH (University Teaching Hospital) and CDH (Cancer Diseases Hospital) where I have had the experience of working in PC, both managements have been supportive towards PC, so it's not a big challenge. (Respondent 6).

And

"The support environment that is now existing with management and the civil service recognising that you need palliative care teams. Not just in terms of doctors but also the fact that they want to implement this palliative care across all health specialties that drives me on." (Respondent 6).

Support from the Palliative Care Alliance Zambia (PCAZ) was also recognised by participants as being instrument in there setting up of the palliative care teams and units.

"The Palliative care Association of Zambia (PCAZ), haaa, because having seen that we were quiet active and vigorous in implementing palliative care, haaa, they provided us with both financial, material, and also some training both at diploma level, like for me I was trained through, haaa, through the same as well as many others at the hospital and in the country. Then, so the PCAZ they provided us with a bit of funding that we were able to use, for training purposes of the hospital staff, haaa, for hosting events like the palliative, world palliative care day as well as sending more of our staffs to Uganda for a training in palliative care." (Respondent 1).

"...I think there I want to say thank you to the Palliative Care Alliance of Zambia, they really did quite a lot of work in training community health workers on palliative care. So, that helped us even and continues to help once it comes to 
identifying patients in the community who would benefit from palliative care service..." (Respondent 4).

"The Palliative Care Association of Zambia, also has recognise our office, haaa, and they even helped us start off the office, they gave us some stationary, they gave us some furniture, and even the computer, and the also gave us the computer and printer for us to use for any work we are doing..." (Respondent 5).

\subsection{Effective Team Work and Delivery Models}

Participants described that team work was an inspiration to practice and implement palliative care services. They also expressed that support and collaborations from other palliative care teams and organisation providing palliative care was quiet instrumental. The presence of an international mentor was reported to have been beneficial. Further all highlighted to have used a hospital based model which included in-patient and out-patient models with an extension into the community through collaborative partnerships.

“Palliative care is not a one man's job, this is something that is done in a multidisciplinary way and you know as I said is not a ones man's job. So most of the support, I mean besides the management support and now coming to the actual work, I mean, we, we have been working in a team..." (Respondent 1).

And

"We do the hospital based palliative care... we take care of them within the hospital facilities... we realize that some may need to be cared from home so we refer them, I mean we discharge them to their home, haaa, through one of the local, haaa, hospice, I mean Home Based care unit..." (Respondent 1).

"...the palliative care coordinator) comes once in a while to the unit to encourage us, and we usually have meetings on Tuesdays to encourage each other and to know on how to take care of our patients who need palliative care. And we've got a few nurses in here too, who have done a short courses in palliative care so at least we consult each other..." (Respondent 2).

"... we are essentially facility based, we looked at our community and considered that we do have outreach services, but we are limited to what those outreach services can do. So we use our outreach, our community health workers to identify patients who will benefit from palliative care...." (Respondent 4).

And

"...We have been fortunate that we are located in an area where we still have operational hospices. Haaa, they are two hospices, Our Lady's Hospice and Mother Theresa Hospice. And we have worked very closely with them. Our capacity to admit patients is very limited. So whenever we have patients who we feel would benefit from not necessarily a hospital admission but still need to be admitted in a supervised setting we have been able to refer patients to them. And they like likewise when they have patients who they need us to attend to they are able to come to us. Haaa, we have a give and take relationship. I think having that kind of support from a lot of partners makes palliative care more sustainable." (Respondent 4). 


\subsection{Personal Development and Motivation to Serve}

Participants highlighted that their desire to provide palliative care services and the satisfaction from the patients and their families motivated the participants to serve. Further, they expressed that their inner drive and passion to serve and to see palliative care services implemented was vital in their work.

"...in as much as most of us did this training because of the love that we had for the subject and the joy that it gave us ah to see I mean, the dying person smile, or the family that is bereaved smile..." (Respondent 1 ).

"I think for me you can't go away from palliative care, the need is there. A lot of our patients present to hospital quiet late. So in terms of what you can do cure wise, a lot of patients come at a point when you cannot achieve cure. So all you can do is try to make the patient as comfortable as possible. So ha, that is a big motivation for me." (Respondent 4).

"...probably the other thing is, it's a new field, there isn't much research that has happened in there. So there is potential for personal growth as well. In terms of research, in terms of career development. It's a route that you can take, so that also is an important aspect." (Respondent 6).

And

"So through my experience of training in PC. It has given me opportunities, haaa, to serve. For example, I have served as vice chair (chairperson) of the Palliative Care Alliance Zambia board. That is an opportunity that would not have come without the training in palliative care." (Respondent 6).

\subsection{Challenges/Barriers}

The theme identified under challenges were: Lack of PC knowledge; lack of essential medication for PC; increased work load/work burden; lack of financial resources; lack of acceptance of PC; lack of PC guidelines; and legal restrictions on the use of opioids.

\subsection{Lack of PC Knowledge}

Study participants reported that PC knowledge was lacking in the health care providers, patients and their families. It was also highlighted that untrained health workers felt uncomfortable to prescribe and use morphine for pain management. They also noted that lack of knowledge resulted in them not being supported by their colleagues as they did not understand the importance of palliative care.

"The challenges that I face, haaa, here where am working, ummm, mostly it's with my staff because of lack of man power, lack of people who've been trained in this, umm, in this case, doctors and nurses. With doctors I can say that maybe the patient is in pain. May be you suggest that maybe you give them morphine, they've got their own reasons why they don't want give morphine, and to start with, the sister in charge herself, she, she doesn't want to order morphine..." (Respondent 2). 
"We still have a lot of people who really don't understand palliative care. To them, just like my understanding before I did the (palliative care) training was something like end of life. So when they are referring a client to you, they normally just want to referrer somebody who is at end of life..." (Respondent 3).

"There are still a lot of challenges, ha, I think the first one is, people don't know what palliative care is. And this is both health care providers and patients themselves." (Respondent 4).

\subsection{Lack of Essential Medication for PC}

Participants reported that inadequate essential palliative care medication such as morphine and laxatives negatively affected effective PC implementation.

"...one of the things we have been trying to do was to get access to morphine for management of moderate to severe pain. So it has been very difficult for us to, to get access to the drug, because of the way the health system is structured..." (Respondent 4).

"... They pharmacy itself here in UTH does not stock enough morphine... they have to reconstitute only so much, so they give me maybe $100 \mathrm{mls}$ and here I am sending this patient home, am asking them to go and manage the pain at home." (Respondent 5).

And

"I want them to give me a supply of 1 month, but they give me $100 \mathrm{mls}$, which is not enough." (Respondent 5).

"...sometimes, not always, but lack of drugs that are important in palliative care especially morphine. If you have morphine, you don't have laxatives. So it becomes difficult to practice in such an environment." (Respondent 6).

\subsection{Work Burden}

Study participants reported that lack of PC knowledge resulted in them not receiving support from their colleagues which led to increased workload or work burden.

"The challenges that I face, haaa, here where am working, ummm, mostly it's with my staff because of lack of man power, lack of people who've been trained in this, umm, in this case..." (Respondent 2).

"The other biggest problem we have, is the work burden, is too much, eeeeh, because right now we don't have enough people to go round the wards to review the patients..." (Respondent 5).

And "...I don't get support because of challenges of work, work load, people are busy, people are tired...” (Respondent 5).

\subsection{Lack of Financial Resources}

Participants said that implementation of PC services was rather difficult because of financial constraint as PC was not included in the institutional budget lines.

"So the other challenge that we had was..... haaa lack haaa.... of finances..... 
for a number of things that we.... we noticed that we needed to do which required money. Among them to prepare pain management forms, haaa.... referral forms and other things included, ehhh, and then when we wanted to follow up some patients... our clients in the community, haaa, that also needed some sort of financing so that is what we... we.... so those were the major major challenges that we experienced with regards to implementing palliative care." (Respondent 1 ).

“...It goes back to people not knowing it. So in terms of allocation, already in a resource limited setting, to get resources allocated to palliative care programs is not easy." (Respondent 4).

"The other thing that we need though it's not a thing that is there is financial support. Financial support programme is not there." (Respondent 6).

\subsection{Lack of Acceptance of PC}

Some participants said that PC had not yet not been appreciated and recognised in Zambia. They reported that, only few palliative care positions were noted to have been recently been created in the civil service.

“...palliative care although has been in Zambia since 2009, it was not really implemented, you know, it has not moved to a level where people have accepted it here in the hospital and in Zambia per se..." (Respondent 5).

"...lack of, haaa, the service having been incorporated into the civil service, only recently has it been introduced. So in the past it has been very difficult to even talk about PC with an establishment it becomes much easier for people to practice." (Respondent 6 ).

\subsection{Lack of PC Guidelines}

Lack of national standard operating procedures, guidelines and treatment protocols for PC were also observed as a challenge in implementing of the service.

"Also for pain management I think that's an area that we have not done very well, so we still have a lot of patients who are in pain when they don't need to be in pain and when they are medicines, drugs and things that we can do, it's just that they are no laid down procedures and protocols for the management of PC cases, it makes you want to do more." (Respondent 4).

"The lack of national guidelines, ok, there is some form of guideline in the Ministry of Health, but it's not clearly defined for you to practice as a palliative care practitioner, ok." (Respondent 6).

\subsection{Legal Restrictions on the Use of Opioids}

Some participants noted that the Zambian law did not allow them to prescribe morphine which negatively affected effective pain control in their patients. Further, lack of national standard operating procedures, guidelines and treatment protocols for PC were also observed as a challenge in implementing of the service. 
"...because of the way the health system is structured, haaa, only doctors can prescribe morphine, haaa, the restrictions on how it can be administered looking at the indirect. So that has been a very big problem for us..." (Respondent 4).

And

"also for pain management I think that's an area that we have not done very well, so we still have a lot of patients who are in pain when they don't need to be in pain and when they are medicines, drugs and things that we can do, it's just that they are no laid down procedures and protocols for the management of PC cases, it makes you want to do more. (Respondent 4).

"...my personal challenge is that, because am a nurse, and here in this country, nurses are not allowed to prescribe morphine..." (Respondent 5).

\subsection{Discussion}

Eleven themes arose from the study as factors influencing implementation of PC, which were broadly classified as facilitators or challenges. Among the facilitators, themes that arose where: training influence; higher level support; effective team work and delivery models; and personal development and motivation to serve. challenges included: lack of PC knowledge; lack of essential medication for PC; increased workload/work burden; lack of financial resources; lack of acceptance of PC; lack of PC guidelines; and legal restrictions on the use of opioids.

\section{Facilitators}

\subsection{Training Influence}

All the respondents mentioned that the training was important because it reduced the knowledge gap as it enabled them to successfully implement PC. This is in relation to Kumar et al. (2011) were they observed that a palliative care training had significant positive impact on the knowledge, attitudes, beliefs and experiences among the participants [24]. They further emphasised that the PC training had a positive impact on PC implementation.

\subsection{Higher Level Support}

Study participants highlighted that because the Zambian government created a few palliative care positions in the civil service establishment they felt motivated and supported. Further, management support at institutional level and by the Palliative care association was reported to have made palliative care implementation easier and feasible. This was also observed by Hasson et al. (2008) and Clark et al. (2007) who stated that good management support played a huge role in the success of their palliative care work thereby positively influencing palliative care implementation [13] [17]. Similarly, Hasson et al. (2008) added that management support made access to resources easier for effective palliative care implementation. Therefore, higher level support was seen as a relevant factor for the success of PC implementation [17]. 


\subsection{Effective Team Work and Delivery Models}

Participants described that team work was an inspiration to practice and implement palliative care services. They also expressed that support and collaborations from other PC teams and organisation providing palliative care was quiet instrumental. This was similar to what was observed by Clark et al. (2007) in South Africa where complete multidisciplinary team allowed practitioners to offer quality palliative care services and support other departments that needed the services [13]. The presence of an international mentor was reported to have been beneficial, as what Hasson et al. (2008) reported that the flexibility and availability of the supervisors for advice encouraged palliative care implementation [17]. All facilities used a hospital based model which included in-patient and out-patient models with an extension into the community through collaborative partnerships with organisations that used home based care and hospice models. Effective team work, collaborations and mentorship showed a positive influence on PC implementation.

\subsection{Personal Development and Motivation to Serve}

Palliative care being a relatively new field it was seen by participants as an area with potential for personal and career development with room for research. Further participants also highlighted that their desire to provide palliative care services and the satisfaction from the patients and their families motivated them to serve. Participants expressed that their inner drive and passion to serve and to see palliative care services implemented was vital in their work. Correspondingly, Clark et al. (2007) underlined that the desire to serve couple with the complete multidisciplinary team gave an extra zeal for practitioners in South Africa to offer quality palliative care services. Hence an inner desire to serve is very relevant for effective PC services [13].

\section{Challenges}

\subsection{Lack of PC Knowledge}

Most of the participants reported that PC knowledge was lacking in the health care providers they were working with, in patients and their families. Untrained health workers felt uncomfortable to use morphine for pain management as they feared addiction. Moreover, their lack of knowledge also resulted in them not supporting their colleagues who were implementing PC. McDermott (2008) research revealed that lack or little education about morphine had hindered effective implementation of palliative care in India [16]. The was also observed by Clark et al. (2007) and Brazil et al. (2010) who reported that lack of palliative care training and its absence in the medical curriculum had impacted negatively on effective implementation of palliative care services [13] [18]. Therefore, inadequate $\mathrm{PC}$ knowledge has a negative effect on the provision of this service.

\subsection{Lack of Essential Medication}

Participants reported that lack of essential palliative care medication especially 
morphine and laxatives negatively influenced their PC implementation. This finding was similar to a study by McDermott (2008) who revealed that opioid accessibility and availability was a constant challenge for palliative care providers [16]. Onyeka (2011) also stated that unavailability of opioids prevented proper management of pain in Nigeria. Essential PC drugs are very important for effective PC implementation [11].

\subsection{Work Burden}

Due to the increase in the increase in the number of patients suffering from life threatening illnesses it was reported that the demand for palliative care services caused increased work load leading to work burden. Additionally, study participants also reported that lack of PC knowledge resulted in them not receiving support from their colleagues which led to work burden. This is in relation to Onyeka (2011) and Grant et al. (2011) who reported that health care workers could not meet up to the overwhelming demands of patients needing PC due increased disease burden [11] [14]. Hence, overburdened health care workers are unlikely to provide effective PC.

\subsection{Lack of Financial Resources}

Inadequate financial support was reported by the participants as a major challenge in their implementation of PC. This was also observed by Stjernsward et al. (2007), Brazil et al. (2010), McDermott (2008) and Onyeka (2011) who reported that the implementation of palliative care services lacked sufficient funding which affected effective PC implementation [2] [11] [16] [18]. It's therefore evident that lack of funding and recognition has a negative effect on PC implementation.

\subsection{Lack of Acceptance for PC}

The study highlighted that PC had not yet been appreciated and recognised in Zambia. It was reported that only recently a few PC positions were included in the civil service. It was observed by Onyeka (2011) that PC was a new specialty in the developing world; hence its acceptance was compromised as it was not included in most of the existing health structures [11]. This was also observed by Goma et al. (2008) who highlighted that palliative care was not commonly practiced in Zambia even when the health care workers were away of it [4]. Therefore lack of acceptance of PC greatly affects PC provision.

\subsection{Lack of PC Guidelines}

Lack of national standard operating procedures, guidelines and treatment protocols for PC were also observed as a challenge in implementing of the service. Similarly, Stjernsward et al. (2007) reported that inclusion of PC in the national health care plan, created opportunities for funding and service deliver models that supports service delivery [2]. Further, Ndungu (2011); Mwangi-Powell (2011); 
Rawlinson et al. (2014); Logie, (2012); Goma et al. 2008; Kalolo, 2010) reported that most countries, Zambia included, had no PC in their national policies, hence causing lack of national PC guidelines [3] [4] [19] [20] [21] [22]. Therefore, lack of national PC guidelines usually results in PC not being included in the national health system.

\subsection{Legal Restrictions on the Use of Opioids}

Palliative care nurses complained that the Zambian law only allowed medical doctors to prescribe opioids such as morphine which negatively affected effective pain control. Likewise, McDermott (2008) revealed that opioid accessibility was a constant challenge for palliative care providers who highlighted stringent government legislation, reluctance to implement simplified narcotics regulations and opiophobia as some of the key barriers to morphine availability [16]. Therefore, legal restrictions especially on opioid use must be taken into consideration to prevent poor PC implementation.

Lack of PC knowledge, inadequate palliative care essential drugs and work burden, came out as a huge challenge. This is because palliative care services were not fully rendered to patients in need as the health care personnel with no knowledge of the benefits of palliative care did not see the need to refer such patients to palliative care trained individuals. This challenge was compounded by lack of essential drugs for a palliative care to use for the patients who accessed the services. Moreover, the palliative care trained personnel were not generally recognised in the country hence they rendered palliative care services in their free time aside from their normal routines, and this turned out to be a work burden to them.

Therefore, the research produced evidence of the importance of palliative care and how it impacts positively on the patients' quality of life. Therefore, provision of palliative care is necessary for all patients with life limiting illnesses. Therefore, all HCWs must be trained in palliative care so that they can have the knowledge enough to identify and refer patients for palliative care at an appropriate time. The trained palliative care personnel at an advanced level must be recognised and employment to be enable them to be able to put hundred percent of their time in palliative care.

\section{Limitations of the Study}

A number of important limitations need to be considered: Firstly, this was a qualitative study and as such it reflects only true meaning as there could be more than one interpretation of the narratives. Secondly, results may be influenced by the researcher's experiences; and thirdly, only participants from three institutions were included in the study, therefore the findings cannot be generalized to other institutions.

\section{Recommendations}

It is recommended that more research and education should be done with a 
concentration on pain management specifically pain management drugs and their effects on the patients. Effective teamwork is recommended as the research revealed that participants needed support for effective implementation of palliative care. Further, there is need for government to create more positions for palliative care specialists if it's to be fully implemented.

\section{Conclusion}

Facilitators and challenges emerged through the data. Among the facilitators where: training influence; higher level support; effective team work and delivery models and personal development and motivation to serve whilst challenges included: lack of PC knowledge; lack of essential medication for PC; work burden; lack of financial resources; lack of acceptance of PC; lack of PC guidelines; and legal restrictions on the use of opioids. Therefore, the study provided evidence of the challenges and facilitators that influence palliative care that specialists experience while implementing PC.

\section{Acknowledgements}

My God the Almighty for the gift of life and my medical career. My dear husband, friend Festus Simuunza and my sons Pascal, Nathanael, Jayson and Miguel Simuunza. Ministry of Health-Cancer Diseases Hospital.

\section{Conflicts of Interest}

The authors declare no conflicts of interest regarding the publication of this paper.

\section{References}

[1] WHO (2014) Global Atlas of Palliative Care at the End of Life.

[2] Stjernsward, J., Foley, K.M. and Ferris, F.D. (2007) The Public Health Strategy for Palliative Care. Journal of Pain and Symptom Management, 33, 486-493. https://doi.org/10.1016/j.jpainsymman.2007.02.016

[3] Logie, D.E. (2012) An Evaluation of a Public Health Advocacy Strategy to Enhance Palliative Care Provision in Zambia. BMJ Supportive \& Palliative Care, 2, 264-269. https://doi.org/10.1136/bmjspcare-2012-000232

[4] Goma, F., et al. (2008) The 2008 Situational Analysis of Palliative Care in Zambia, PCAZ.

[5] Street, A. and Blackford, J. (2001) Communication Issues for the Interdisciplinary Community Palliative Care Team. Journal of Clinical Nursing, 10, 643-650. https://doi.org/10.1046/j.1365-2702.2001.00549.x

[6] Murray, S., Boyd, K., et al. (2008) Implementing a Service User's Framework for Cancer Care in Primary Care: An Action Research Study. Family Practice Journal, 25, 78-85. https://doi.org/10.1093/fampra/cmn011

[7] Kulkarni, P.D. (2011) Hospital-Based Palliative Care: A Case for Integrating Care with Cure. Indian Journal of Palliative Care, 17, S74-S76.

https://doi.org/10.4103/0973-1075.76248

[8] Central Intelligence Agency (2014) World Factbook 2014. 
https://www.cia.gov/library/publications/the-world-factbook/geos/za.html

[9] Cancer Diseases Hospital (2014) Annual Report for 2014. Lusaka.

[10] Walliman, N. (2011) Your Research Project. 3rd Edition, Sage Publications, London.

[11] Onyeka, T.C. (2011) Palliative Care in Enugu, Nigeria: Challenges to a New Practice. Indian Journal of Palliative Care, 17, 131-136. https://doi.org/10.4103/0973-1075.84534

[12] Ntizimira, C.R., Nkurikiyimfura, J.L., Mukeshimana, O., Ngizwenayo, S., Mukasahaha, D. and Clancy, C. (2014) Palliative Care in Africa: A Global Challenge. Ecancermedicalscience, 8, 493. https://doi.org/10.3332/ecancer.2014.493

[13] Clark, D., Wright, M., Hunt, J. and Lynch, T. (2007) Hospice and Palliative Care Development in Africa: A Multi-Method Review of Services and Experiences. Journal of Pain and Symptom Management, 33, 698-710. https://doi.org/10.1016/j.jpainsymman.2006.09.033

[14] Grant, L., Downing, J., Namukwaya, E., Leng, M. and Murray, S.A. (2011) Palliative Care in Africa since 2005: Good Progress, But Much Further to Go. BMJ Supportive \& Palliative Care, 1, 118-122. https://doi.org/10.1136/bmjspcare-2011-000057

[15] Basu, A. (2013) Palliative Care in Low- and Medium-Resource Countries. The Cancer Journal, 19, 410-413. https://doi.org/10.1097/01.PPO.0000434392.18811.3c

[16] McDermott, E. (2008) Hospice and Palliative Care Development in India: A Multi-Method Review of Services and Experiences. Journal of Pain and Symptom Man agement, 35, 583-593. https://doi.org/10.1016/j.jpainsymman.2007.07.012

[17] Hasson, F., Kernohan, W.G., Waldron, M., Whittaker, E. and Mclaughlin, D. (2008) The Palliative Care Link Nurse Role in Nursing Homes: Barriers and Facilitators. Journal of Advanced Nursing, 64, 233-242. https://doi.org/10.1111/j.1365-2648.2008.04803.x

[18] Brazil, K., Kassalainen, S., Ploeg, J. and Marshall, D. (2010) Moral Distress Experienced by Health Care Professionals Who Provide Home-Based Palliative Care. Social Science \& Medicine, 71, 1687-1691. https://doi.org/10.1016/j.socscimed.2010.07.032

[19] Ndungu, H. (2011) Palliative Care: What Approaches Are Suitable in Developing Countries? British Journal of Haematology, 154, 728-735. https://doi.org/10.1111/j.1365-2141.2011.08764.x

[20] Mwangi-Powell, F. (2011) Palliative Care in Africa: An Overview. Africa Health, 33, 19.

[21] Rawlinson, F.M., Gwyther, L., Kiyange, F., Luyirika, E., Meiring, M. and Downing, J. (2014) The Current Situation in Education and Training of Health-Care Professionals across Africa to Optimise the Delivery of Palliative Care for Cancer Patients. Ecancermedicalscience, 8, 492. https://doi.org/10.3332/ecancer.2014.492

[22] Kalolo, D. (2010) Acceptability of Pain Assessment Tools by Doctors and Nurses at University Teaching Hospital and Cancer Diseases Hospital. MSc Dissertation, UNZA, Lusaka.

[23] Aveyard, H. (2010) Doing a Literature Review in Health and Social Care. A Practical Guide. 2nd Edition, Open University Press, Maidenhead.

[24] Kumar, S.P., Jim, A. and Sisodia, V. (2011) Effects of Palliative Care Training Program on Knowledge, Attitudes, Beliefs, and Experiences among Student Physiotherapists: A Preliminary Quasi-Experimental Study. Indian Journal of Palliative Care, 17, 47-53. https://doi.org/10.4103/0973-1075.78449

[25] McDermott, E., et al. (2008) Hospice and Palliative Care Development in India: A Multimethod Review of Services and Experiences. Journal of Pain and Symptom 
Management, 35, 583-593. https://doi.org/10.1016/j.jpainsymman.2007.07.012

[26] African Palliative Care Association (2010) Palliative Care: A Handbook of Palliative Care in Africa. African Palliative Care Association, Kampala, Uganda.

[27] Becker, G., Hatami, I., Xander, C., Dworschak-Flach, B., Olschewski, M., Momm, F., et al. (2011) Palliative Cancer Care: An Epidemiologic Study. Journal of Clinical Oncology, 29, 646-650. https://doi.org/10.1200/JCO.2010.29.2599 